\section{Psychotic Disorder}

Paul Newman ${ }^{1}$ and Ana Mills ${ }^{2}$

${ }^{1}$ Department of Medical Psychology and

Neuropsychology, Drake Center, Cincinnati, OH, USA

${ }^{2}$ Department of Physical Medicine \&

Rehabilitation, Virginia Commonwealth

University, Richmond, VA, USA

\section{Synonyms}

Psychosis

\section{Definition}

A disorder characterized by one or more of the following abnormal behaviors: delusions, hallucinations, disorganized thinking, disorganized or abnormal motor functioning, and negative symptoms. The individual often lacks insight into their condition. Psychotic disorders have diverse causes. Etiologies may include disease states such as schizophrenia, delirium, metabolic disorders, genetic disorders, medical disorders (e.g., tumors, epilepsy), and drug/medication reactions.
The neurobiology and neuropsychology of more common psychotic conditions such as schizophrenia are well documented. Structural lesions have also been associated with rare psychotic conditions such as reduplicative paramnesia.

\section{Cross-References}

$\checkmark$ Psychosis

- Reduplicative Paramnesia

- Schizophrenia

\section{References and Reading}

American Psychiatric Association. (2013). Diagnostic and statistical manual of mental disorders (5th ed.). Arlington: American Psychiatric Association Publishing.

Ardila, A. (2016). Some unusual neuropsychological syndromes: Somatoparaphrenia, akinetopsia, reduplicative paramnesia, autopagnosia. Archives of Clinical Neuropsychology, 31(5), 456-464.

Kleiger, J. H., \& Khadivi, A. (2015). Assessing psychosis: A clinician's guide. New York: Taylor \& Francis.

Schaefer, J., Giangrande, E., Weinberger, D. R., \& Dickinson, D. (2013). The global cognitive impairment in schizophrenia: Consistent over decades and around the world. Schizophrenia Research, 150(1), 42-50. 